\title{
Simulation of Crop Evaportranspiration Based on BP Neural Network Model and Grey Relational Analysis
}

\author{
Liang Ma, Feng Liu, Liangliang Chen, Ming Hong \\ School of Water Resources \& Civil Engineering, Xinjiang Agricultural University, Urumqi City, Xinjiang \\ Province, China
}

\begin{abstract}
Crop evaportranspiration was studied with measured data of Kongque river irrigation district in Xinjiang Province based on application of BP neural networks, a sensitivity analysis about crop evaportranspiration was conducted according to each input factor by using default factor method, and the grey relational analysis method was applied to certify the results. The results showed that the artificial neural networks model could express quantitatively the response relationship between crop evaportranspiration and various factors with sufficient high accuracy. Soil moisture and solar radiation were the main sensitive factors for soil water-salt dynamic in this irrigation district, the interaction amongst various factors formed coupling relationship under the complicated condition. The grey relational analysis method could further verify the sensitivity degree amongst various factors. The combination of the above methods provides feasible method for analyzing the rules of crop water comsumption during crop growing season, and it is complement and perfection for the traditional research methods of crop evaportranspiration.
\end{abstract}

Index Terms: arid zone;BP neural networks; crop evaportranspiration; sensitiveness factors; grey relational analysis

(C) 2012 Published by MECS Publisher. Selection and/or peer review under responsibility of the Research Association of Modern Education and Computer Science.

\section{Introduction}

Evapotranspiration water through the soil-plant-atmosphere system of continuous transmission, air, soil, crops[1].At present, the calculation of crop evapotranspiration evaporation many ways, can be broadly grouped into test formula, water vapor diffusion method, the energy balance method and synthesis method, etc. several categories.Among the most widely used energy balance method, the basic idea is: the evapotranspiration process as energy consumption, calculated by balancing the energy consumption calculated and then converted into energy water that crop water requirements.However, in the above study on the impact of changes in evapotranspiration and the sensitivity of the sensitive factors, little is currently involved in discussions.In this

* Corresponding author.

E-mail address: xjml1999@163.com 
paper, the conditions in arid areas of crop evapotranspiration during the growing season changes in the complexity of the basis of previous research, the use of BP neural network technology to build an evapotranspiration model, describes the combination of factors on crop evapotranspirationthe impact test method using the default factor analyzed the impact of factors on the sensitivity of evapotranspiration, and the gray relational analysis factors on the impact of a comprehensive order to test the neural network accuracy of the default factor method, also completedGrey self-examination.

\section{BP Neural Network Model}

Analysis of ecological factors such as weather and soil on evapotranspiration of the main methods for three class .1) The correlation and multiple linear regression method, this method easy and intuitive[2,3], but even taking into account the polynomial regression and interaction of various factors, the effect of predicted and actual conditions are often not very consistent,analyzed the main reasons for crop evapotranspiration complex relationship between factors, a simple multiple regression approach does not reflect the relationship between them.2)Joint multivariate multiple regression analysis methods such as principal component analysis and factor analysis[4], this method is Method 1) improvements to a certain extent, reduce the interaction between the factors on the ultimate goal of factor[5], but also to reflect the qualitative relationship between the various relevant factors, but multiple linear regression methods are not completely describe the linear law of agriculture there are still many defects[6].3)Non-linear, non-parameter calculation methods, such as classification and regression tree method, boundary line analysis and artificial neural network analysis[7].

\subsection{Breifness on BP neural network}

BP neural network is the most widely used is the most mature development of a neural network.It is the input layer, one or more of the hidden layer and output layer connection from each of its layer and by the many artificial neurons (nodes), with a layer of neurons within the adjacent layers only, and the next levelThe neuronal connections.BP network connection weights between the learning process in the network continue to be amended so that the input layer and hidden layer, hidden layer and output layer of rights between the two groups to achieve learning network composed of input vectors and output samplesmapping relationship between the specific vector, the distribution of power reflects the components of the input characteristics of the input vector strength of the share distribution. When the study sample provided to the network, the activation values of neurons from the input layer through the middle layer to output layer spread in the output layer of the neural network input received response.After this, the desired output by reducing the output error and the actual direction of the middle layer from the output layer by layer by layer correction for the connection weights, and finally back to the input layer.Connection weights of each adjusting the standard error back propagation algorithm or cumulative error back propagation algorithm.Neural network by modifying the weights of each layer, and the error decreasing, when the error reaches pre-determined requirements, the network learning and training process is completed.

\subsection{The transfer function and weight}

BP neural network training is continuously adjusted by the weights of each layer, making the desired output value and actual value of the error to a minimum.Model of the initial value of random weight assignment, and the input layer and hidden layer transfer function is applied as follows Sigmoid function, the hidden layer and output layer transfer function using the linear function:

$$
f(x)=\frac{1}{1+e^{-x}}
$$


The change interval $[0,1]$.

\subsection{Data Preprocessing}

In the network learning process, to facilitate training, to better reflect the relationship between various factors, must be pre-sample data. General network output vectors of the sub-values should be in $[0,1]$. To a large input neuron activation function fell on major regional gradient of the input vector is also taken of each component $[0,1]$ features is good. Therefore, before training network, the input data normalization, processing is as follows:

$$
x_{n o r m}=\frac{x_{i}-x_{\min }}{x_{\max }-x_{\min }}
$$

Where: $x_{n o r m}$-normalized value for the sample, $x_{\max }$-maximum value for the sample, $x_{\min }$-minimum value for the sample, $x_{i}$-the sample values.

\section{Artificial Neural Network Model}

\subsection{Input, output factors pre-selection and training samples}

The actual situation for irrigation, the establishment of the crop evapotranspiration dynamic BP network model, considering various factors, select the soil moisture content, average daily temperature, daily average wind speed, daily average relative humidity, daily solar radiation as the input factors, crop evapotranspirationAs the amount of output variables, a comprehensive reflection of the major factors on the impact of water and salt.Sample data from the irrigation of crops during the growing observational data.Among them, the determination of the soil layer averaged related indicators, each layer of $0.1 \mathrm{~m}$ as measured depth of $0.8 \mathrm{~m}$.All samples were participating in the network training and testing.

Neural network data pre-processing, have a crucial impact on the network. The foregoing factors on the changes in the value of difference between the larger, and the dimensions are not the same, the use of actual data will seriously affect the learning speed and accuracy, it can not be directly used for training; input factors of the pre-existing in the paston the basis of the data, taking into account in the next phase of changes in soil water and salt, so the input and output data standardization certain prediction of space left, the training methods used normalized to the actual data processing $[0,1]$.

\subsection{Model Training and Testing}

In this study, 90 groups evapotranspiration input and output samples.To make the model has a generalization, select the crop growth period of the 70 groups of samples as the evapotranspiration of the training samples.Studies have shown that three-layer BP network can approximate any rational function, this three-layer BP network selected, the hidden layer to hyperbolic S-function $f(x)=1 /\left(1+e^{-x}\right)$ as the transfer function of BP neural network,linear function of the output layer to the input and output samples for training.After repeated adjustments hidden layer nodes, and ultimately determine the network topology is 5:11:1, the model in the training process showed good performance of error convergence.

With 20 groups not involved in the training input and output samples to test the model.The results show that this network model has high accuracy, the average evapotranspiration test error of $0.24 \mathrm{~mm}$, characterized by a combination of factors can crop evapotranspiration under changes to meet the crop evapotranspiration during the growing season forecast requirements. 


\section{Evapotranspiration Model Sensitivity Analysis}

\subsection{The default factor test method}

The impact of crop evapotranspiration for a sensitive factor for the default factor artificial neural network model test, that is, by default all input factors model of the neural network and testing, according to the test error and test error of the whole factor modelThe default ratio of Ri to determine the size factor on the output sensitivity of the response factors.

$$
\mathrm{R}_{\mathrm{i}}=\mathrm{RMSE}_{\mathrm{i}} / \mathrm{RMSE}
$$

Where: $\mathrm{R}_{\mathrm{i}}$-sensitivity index; $\mathrm{RMSE}_{\mathrm{i}}$-the model testing errors without factor $\mathrm{i}$; RMSE-full factorial model testing errors.If $R M S E_{i}>R_{M S E}$, said the factor $i$ more sensitive than the factor $j$.

\subsection{Results of the sensitivity factor analysis}

Evapotranspiration model will be established with the 5 default input factors one by one, the establishment of four factors evapotranspiration neural network model.To make 4 and 5 factor model factor model is comparable, in the modeling process used in the training samples, test samples equivalent to 5 factor model, the same network using BP learning algorithm for simulation.

By default factor test method, the results showed that (see Table 1): 4 factor model test error compared with the five factor model have different levels of increase $(R>1)$, shows the input factors on evapotranspiration volume different degrees.

Table 1. Default factor artificial neural network model test results

\begin{tabular}{ccccc}
\hline factor & $\begin{array}{c}\text { Average } \\
\text { error/mm }\end{array}$ & $\begin{array}{c}\text { Maximum } \\
\text { error/mm }\end{array}$ & $\boldsymbol{R}$ & order \\
\hline 5 factors & 0.24 & 1.14 & & \\
Soil moisture & 1.25 & 3.92 & 5.21 & 1 \\
temperature & 0.57 & 0.89 & 2.38 & 3 \\
Wind speed & 0.31 & 0.63 & 1.29 & 4 \\
$\begin{array}{c}\text { Relative } \\
\text { humidity }\end{array}$ & 0.26 & 0.55 & 1.08 & 5 \\
Solar radiation & 0.98 & 3.46 & 4.08 & 2 \\
\hline
\end{tabular}

On the degree of evapotranspiration sensitivity to five factors: soil moisture>solar radiation>average temperature > average wind speed $>$ average relative humidity.

It can be seen, the soil moisture on the sensitivity of crop water consumption index of the largest of its $\mathrm{R}$ value is 5.21. Shows that the main irrigation crop evapotranspiration influenced by soil moisture, soil moisture is important to characterize the physical properties of the soil parameters are precipitation, canopy interception, plant transpiration, soil evaporation, surface runoff and underground seepage, and otherthe result of factors that can directly affect the crop growth is good or bad, especially large-scale irrigation in the arid inland, the crop water requirement is very huge, in the actual process with particular attention to irrigation, soil moisture changes. 
Sensitivity index of solar radiation is large, $\mathrm{R}$ is 4.08 . Shows that the irrigated crop evapotranspiration influenced by solar radiation. In the process of crop evapotranspiration, solar radiation is the main energy and the increase of temperature to influence through the photosynthesis of crops, thereby affecting the crop evapotranspiration.

Temperature determines the speed of diffusion of water vapor and how much capacity to accept water vapor, atmospheric temperature and humidity directly affect the stratification gradient, thereby affecting soil evaporation. High temperature, the surface of the saturated vapor pressure big, big difference between unsaturated and easy evaporation. Surface air temperature determines the vapor pressure of the size and level of activity of water molecules, the surface temperature is high, the water molecular motion energy, soil evaporation rate is large.

The size of wind speed, the performance of the role of turbulent diffusion in its strength and speed of wet and dry air exchange, the impact of evapotranspiration is the main factor. No wind, the surface mainly by molecular diffusion of water vapor, water vapor pressure decreased slowly, the saturation difference is small, so soil evaporation is slow. When the wind, turbulence enhanced surface wind and turbulent water vapor into the vast space spread rapidly, the surface vapor pressure rapidly decreases, larger saturation deficit, soil evaporation accelerated. The greater the wind speed, the more intense turbulence, wet and dry faster air exchange, so the soil evaporation is also greater.

Relative humidity is the actual vapor pressure in the air under the current temperature the ratio of the saturated vapor pressure, which reflects the degree of air saturation. Therefore, the relative humidity of soil moisture in size to reflect the speed of outward diffusion and exchange. When the relative humidity, the water slowly outward diffusion and exchange, evapotranspiration is small; hours when the relative humidity, soil moisture outward diffusion and exchange of quick, large crop evapotranspiration.

Summing up the sensitivity factor of each analysis, the 5 factors on the irrigated crop evapotranspiration response to varying degrees, soil moisture and solar radiation sensitivity is stronger than the weak sensitivity to other factors, inactual irrigation, various factors should be considered on the impact of crop evapotranspiration for irrigation unified management and control to provide a scientific basis.

\section{Grey Analysis on Crop Evapotranspiration}

\subsection{Principles of gray relational analysis}

Grey relational analysis theory is a new factor analysis method, it is mainly through the sequence of system data to compare the geometric relationship between the factors to analyze the system, the relationship between the degree. Reference sequence set $Y_{0}(k)$, as compared the number of $Y_{i}(k), i=1,2,3, \ldots, m$, sequence length $N$, of the problem is to determine $Y_{i}(k)$ and $Y_{0}(k)$ the degree of close that seek $Y_{i}(k)$ and $Y_{0}(k)$ sequence correlation. In order to ensure correct results of the analysis, first of all raw data should be normalized. In this paper, standardization of transformation, the new data series thus obtained $\mathrm{x}_{0}(\mathrm{k})$ and $\mathrm{x}_{\mathrm{i}}(\mathrm{k})$ is the standard sequence. Correlation coefficient is defined as[8]:

$$
\xi_{i}(k)=\frac{\min _{i \in m} \min _{k \in n}\left|x_{0}(k)-x_{i}(k)\right|+\rho \max _{i \in m} \max _{k \in n}\left|x_{0}(k)-x_{i}(k)\right|}{\left|x_{0}(k)-x_{i}(k)\right|+\rho \max _{i \in m} \max _{k \in n}\left|x_{0}(k)-x_{i}(k)\right|}
$$

Where: $\xi_{\mathrm{i}}(\mathrm{k})$-relative difference between $\mathrm{x}_{0}$ and $\mathrm{x}_{\mathrm{i}} ; \rho$-resolution ratio, taking value generally between 0 to 1 , generally taken $\rho=0.5$.

With the correlation coefficient formula, based on gray relational space above, the correlation is calculated as follows: 


$$
r_{i}=\frac{1}{n} \sum_{k=1}^{n} \xi_{i}(k)
$$

\subsection{Grey relational analysis sensitive factor}

Neural network learning algorithm used here all the 90 groups in the crop evapotranspiration as a comparison series of input samples, output samples as reference sequence for the gray relational analysis. Using (4) and (5), calculated (see Table 2).

Table 2. relational degree of crop evapotranspiration and each factor

\begin{tabular}{cccc}
\hline number & factor & relational degree & order \\
\hline 1 & Soil moieture & 0.9215 & 1 \\
2 & temperature & 0.8041 & 3 \\
3 & Wind speed & 0.7769 & 4 \\
4 & Relative humidity & 0.6938 & 5 \\
5 & Solar radiation & 0.9037 & 2 \\
\hline
\end{tabular}

Table 2 shows the various factors on evapotranspiration of the incidence order is: $r_{1}>r_{5}>r_{2}>r_{3}>r_{4}$, sort results and sensitivity consistent with the results of factor analysis. Thus proving that the BP artificial neural network model of sensitive factor analysis is correct, can be a good quantitative description of the evapotranspiration and response relationship between the influencing factors, but also completed a self-test of gray relational analysis.

\section{Conclusion}

For the evapotranspiration change complex characteristics during the crop growth, the paper will be the introduction of BP neural network simulation of evapotranspiration and forecasting in arid inland areas of crop evapotranspiration studies; and through the establishment of the artificial neural network model, using The default factor test method of gray relational analysis sensitivity of evapotranspiration derived factor:

1) Select the soil moisture content, average daily temperature, daily average wind speed, daily average relative humidity, daily solar radiation as the input factors, crop evapotranspiration as output variables, the evapotranspiration of the BP neural network model, the model network Topology is 5:11:1, the test of the model evapotranspiration error of average test $0.24 \mathrm{~mm}$, has high accuracy.

2) The amount of irrigated crop evapotranspiration and soil moisture on the sensitivity of strong solar radiation, the changes are mainly affected by two factors; and less sensitive to other factors, but in the actual operation of irrigation, other factors also can not be ignored, should be taken into account various factors on the impact of crop evapotranspiration.

3) The sort of gray relational analysis is consistent with the sensitivity factor analysis to further validate the default factor sensitivity of test method accuracy factor, but also completed a self-test of gray relational analysis.

The above method is used in large-scale irrigation in the evaluation of evapotranspiration is feasible, is the traditional method of crop evapotranspiration in the supplementary and improvement of the irrigation water resources control provides a more accurate scientific basis. 


\section{Acknowledgment}

This research work was supported by the Pre-funded Project of Xinjiang Agricultural University(XJAU200921); Autonomous Higher School Research Projects(XJEDU2010S14); Cotton Project Research Center, Ministry of Education, PRC.We give the most heartfelt thanks to the reviewers for their great contributions on the early manuscript.

\section{Reference}

[1] Shang Songhao, Mao Xiaomin, Lei Zhidong. "Winter wheat field moisture forecast BP neural network model,” Journal of Hydraulic Engineering,vol. 33,no. 4,pp.60-63, April 2002.(in Chinese)

[2] Sollich P, Krogh A. Learning with Ensembles: How Over-fitting Can Be Useful. Advances in Neural Information Processing Systems, Denver, CO, Cambridge, MA: MIT Press, 1996.

[3] Pokrajac D, Obradovic Z. "Neural network-based software for fertilizer optimization in precision farming,” Neural Networks, vol. 40,no. 3,pp. 2110-2115, March 2001.(in Chinese)

[4] Joannis N Daliakopoulos, Pauline Coulibaly, Joannis K Tsanis. "Groundwater level forecasting using artificial neural networks,” Journal of Hydrology, vol. 309,no. 7,pp.229-240,July 2005.

[5] Emery Coppola Jr, Mary Poulton, Emmanuel Charles. "Application of artificial neural networks to complex groundwater management problems,” Natural Resources Research, vol. 12,no. 4,pp.303-320,April 2003.

[6] Beaudeau, P. Beaudeau, T. Leboulanger, et al. "Forecasting of turbid floods in a karstic drain using an artificial neural network,” Ground Water, vol. 39,no. 1,pp.109-119,January 2001.

[7] Liu Shuwen, Wang Qingwei, He Dongjian, et al. "Grape disease diagnosis system based on fuzzy neural network,” .Transactions of the CSAE, vol. 22,no. 4,pp. 66-69,April 2006.(in Chinese)

[8] LIU Sifeng, Xie Naiming. Grey system theory and its application. Beijing:Science Press, 2008.(in Chinese) 